Technical note

\title{
Practical active control of cavity noise using loop shaping: Two case studies
}

\author{
Sang-Myeong Kim*, Joao A. Pereira, Vicente Lopes Jr., Antonio E. Turra, Michael J. Brennan \\ Department of Mechanical Engineering, UNESP-Sao Paulo State University, Ilha Solteira 15385-000, SP, Brazil
}

\section{A R T I C L E I N F O}

\section{Article history:}

Received 20 September 2016

Received in revised form 4 December 2016

Accepted 9 December 2016

Available online 10 February 2017

\section{Keywords:}

Noise control

Loop shaping

Second order filters, optimal control

Robust control

\begin{abstract}
A B S T R A C T
A simple loop shaping technique is applied to design an optimal, robust feedback controller to reduce the interior noise of an acoustic cavity. It is a data-based technique that uses the measured plant response to tune the parameters of a fixed-structure controller in a graphical way. The two cases studied are narrowband noise control in a small cavity and broadband noise control in a long duct. Each control system consists of a microphone, a loudspeaker, and a controller connecting the two transducers that are further collocated. The fixed-structure of each controller should be chosen ahead of loop shaping and is determined in this paper solely based on the Nyquist plot of each plant measured. It turns out that a single band (high) pass filter of second order is suitable for the narrowband (broadband) noise control case considered. It is finally demonstrated with experiments that the technique is practical and a second order filter can be effectively used for active control of cavity noise in a single narrow or broad frequency band.
\end{abstract} (c) 2016 Elsevier Ltd. All rights reserved.

\section{Introduction}

Active noise control is a method to reduce acoustic noise by active means such as using loudspeakers. Low frequency noise is of major concern as this noise is difficult to reduce by conventional passive means such as using sound absorbing materials. Since it has such a distinctive application area, it has attracted enormous interest over far more than a half-century. Lueg as early as in 1930s proposed an idea of feedforward control that uses a superposition principle to cancel noise [1]. Olson and May in 1950s then proposed an idea of feedback control to build a very low impedance device [2]. Their ideas have been extensively exploited during the past several decades particularly since the advent of cheap microprocessors in 1980s. Although there are a large number of publications on this subject $[3,4]$, few are concerned with experimental implementations that use feedback control. For example, see [5-10]. One reason for this may be that feedback control is generally more involved in design since it has to ensure stability and robustness. However, there are many practical cases where feedback control is unavoidable because information of the primary noise sources as well as their transfer path characteristics, which feedforward control requires for an implementation, is often unavailable. Hence, more feedback control studies and new data are in need in the field of practical active noise control, i.e., active

\footnotetext{
* Corresponding author.

E-mail address: smkim123@hanmail.net (S.-M. Kim).
}

noise control of such practical cases by practical methods for practical implementations.

The paper presented here reports two experimental case studies that use feedback methods for practical active noise control. The first case is narrowband low frequency noise control in a small cavity, and the second case is broadband low frequency noise control in a long duct. A single-input-single-output control system is constructed in each case using a single microphone and a single loudspeaker. A simple loop shaping technique, similar to that in classical feedback control [11], is then applied to design the controller that connects the microphone and the loudspeaker. As the fixed-structure filter of the controller (i.e., the compensator in classical terms), a single filter of second order is commonly employed for both cases studied. This is because it has been known in active vibration control that this order of filter is effective for controlling vibration in a single frequency band [12-20]. Active vibration control is similar to active noise control in that they are both disturbance rejection control. Thus, those design techniques and rules established for active vibration control are extensively employed in this paper.

Each control filter above can thus be designed under an optimal, robust design framework based on Nyquist robustness criterion, which has been widely used in both active noise $[6,9,10]$ and vibration [15-20] control. The particular loop shaping (i.e., design) method employed is then a data-based technique, for example see [20], that uses the measured plant response to tune the parameters of the fixed-structure candidate controller in a graphical way 
by repeatedly plotting the open loop frequency response until a desirable shape is obtained in terms of both performance and robustness. Thus, no (or minimum) knowledge is required regarding the physics involved, the mathematics on modeling and optimization, and the formalism of modern robust control theory. Instead, elementary knowledge on Bode and Nyquist plots is sufficient for the loop shaping. The control filter designed under this framework is then an optimal controller that is also robust in the sense of Nyquist robustness criterion.

There are other design methods such as, those LQG and $H_{\infty}$ control tools that often require explicit mathematical models of the plant as well as measurable and immeasurable uncertainty $[21,22]$. There is further an IMC (internal model control) technique that transforms the feedback control architecture into a feedforward one so as to give a convex optimization problem involving an FIR (finite impulse response)-type control filter [23]. However, the simple data-based technique described above is preferred in this paper as it is readily applicable, easier to use, and more intuitive and practical for the cases studied. By shaping a control filter of second order, it is finally demonstrated with experiments that more than $40 \mathrm{~dB}$ is reduced for narrowband noise in the first case while the first eight acoustic modes are simultaneously reduced in the second case.

The paper is organized as follows. After this introduction, the two cases studied are described in Section 2. The optimal, robust design framework employed is then presented in Section 3 in equation form by slightly adapting both classical [11] and modern $[21,22]$ robust control theory. Its implications are also discussed together with introducing the room for uncertainty as a robustness measure against immeasurable uncertainty. Section 4 gives some rules and guidelines of loop shaping when second order filters are used as fixed-structure controllers. In Section 5, the control filters for the two cases are actually designed and the experimental data obtained are finally displayed. The paper is closed in Section 6 with some conclusions.

\section{Two plants}

Two acoustic plants are of concern. The first plant is a small cavity as shown in Fig. 1. There are two loudspeakers of the same type (Peerless ${ }^{\circledR}$ P835025), one of which is for exciting the cavity and the other is for controlling it. Each loudspeaker driver was installed in a wooden cabinet (thickness: $15 \mathrm{~mm}$, exterior dimensions: $30 \mathrm{~cm} \times 30 \mathrm{~cm} \times 30 \mathrm{~cm}$ ) packed partly by a piece of sound absorbing foam (thickness $5 \mathrm{~cm}$ ). There are also two $1 / 2^{\prime \prime}$ microphones of the same type (RFT ${ }^{\circledR}$ MV201) located near the diaphragm centers of the two loudspeakers, respectively. One of these is merely for monitoring noise and the other is for controlling by feeding the measured signal $p_{2}$ back to the control loudspeaker

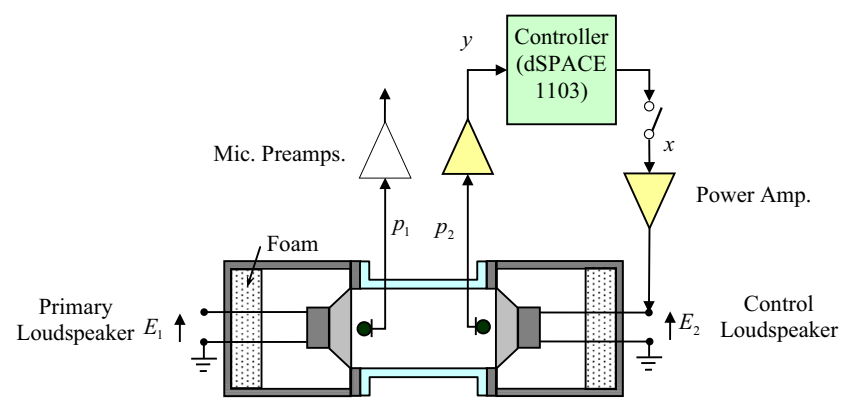

Fig. 1. Narrowband noise control in a small cylindrical cavity (inner diameter $17.5 \mathrm{~cm}$, length $27.2 \mathrm{~cm}$ ) made of acryl (thickness $5 \mathrm{~mm}$ ). through a microphone preamplifier ( $\mathrm{RFT}^{(}{ }^{\circledR} 00$ 023), a controller, and an audio power amplifier $\left(\mathrm{MMF}^{\circledR}\right.$ LV103). The controller platform used was a DSP prototyping machine (dSPACE 1103) running at a sampling frequency of $32 \mathrm{kHz}$. The control filter designed later in this paper was implemented in discrete form within the machine. The plant here resembles a blocked human ear in the acoustical sense $[6,10]$ and of interest is controlling the sub-resonance frequency noise below the fundamental natural frequency (654 Hz: measured) of the cavity. Such noise is largely responsible for the occlusion effects in hearing instruments [24].

The second plant is a long duct as shown in Fig. 2. A sheet of fabric (thickness $1 \mathrm{~cm}$, length $60 \mathrm{~cm}$ ) was rolled and placed inside the duct to give some acoustic damping. The transducers and the feedback control circuit used are exactly the same as those in the first plant, except the location of the control microphone. This microphone was not positioned at the center but an off-center at a nodal point (searched experimentally) of the first radial acoustic mode of the cylindrical duct in order to avoid measuring this mode [25]. This was purposely done because this mode was not a target mode for control so that it is always desirable to exclude its effect from the measurement stage. The plant here resembles a simple acoustic waveguide and of interest is controlling one-dimensional modes far below the cutoff frequency $(2270 \mathrm{~Hz}$ : measured) of the waveguide. Controlling multiple modes is thus of concern across a broad frequency range. A similar task has been successfully conducted for a vibration beam [17]. This is an extension to an acoustic system.

Each plant is the path from the power amplifier input (denoted by $x$ in Fig. 1) to the control microphone preamplifier output (denoted by $y$ ), while the loop is disconnected. The input and output signals were simultaneously monitored by a frequency analyzer (not shown) to produce the frequency response function (FRF). Fig. 3 shows the measured plant response in the small cavity, where both Bode and Nyquist plots are displayed. The sharp peak at $654 \mathrm{~Hz}$ in Fig. 3(a) is due to the fundamental acoustic mode. For a demonstration purpose, the control target chosen was the narrowband noise at around $35 \mathrm{~Hz}$ indicated by the symbol ' $\nabla$ '. This corresponds to the small cardioid-shaped locus located largely in the 'right half of the complex plane in Fig. 3(b). The task here is to design an optimal, robust controller for this and a single filter of second order will be used as the controller. Fig. 4 then shows the measured plant response in the long duct in the same form as Fig. 3, but in an extended frequency range up to $3 \mathrm{kHz}$. For a demonstration purpose, the control target chosen was the broadband noise up to about $1.3 \mathrm{kHz}$ containing the first eight acoustic modes. They correspond to those several circular loci in the lower half' of the complex plane in Fig. 4(b). The task is again to design an optimal, robust controller for this and a single filter of second order will also be used as the controller.

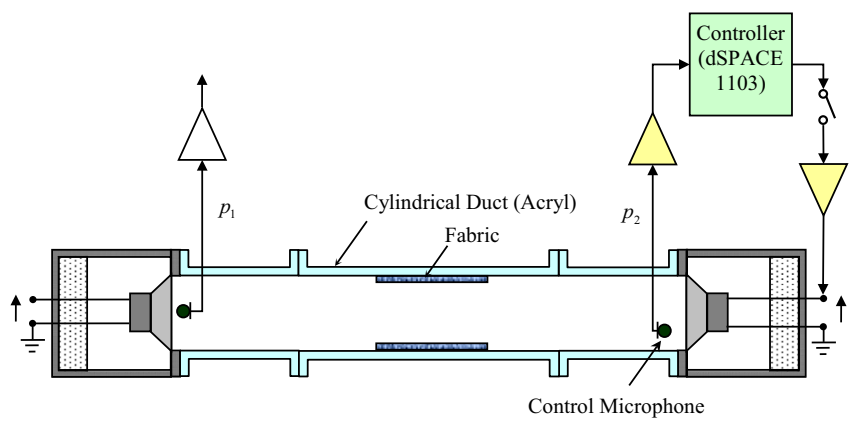

Fig. 2. Broadband noise control in a long cylindrical duct (inner diameter $17.5 \mathrm{~cm}$, length $111 \mathrm{~cm}$ ) made of acryl (thickness $5 \mathrm{~mm}$ ). 

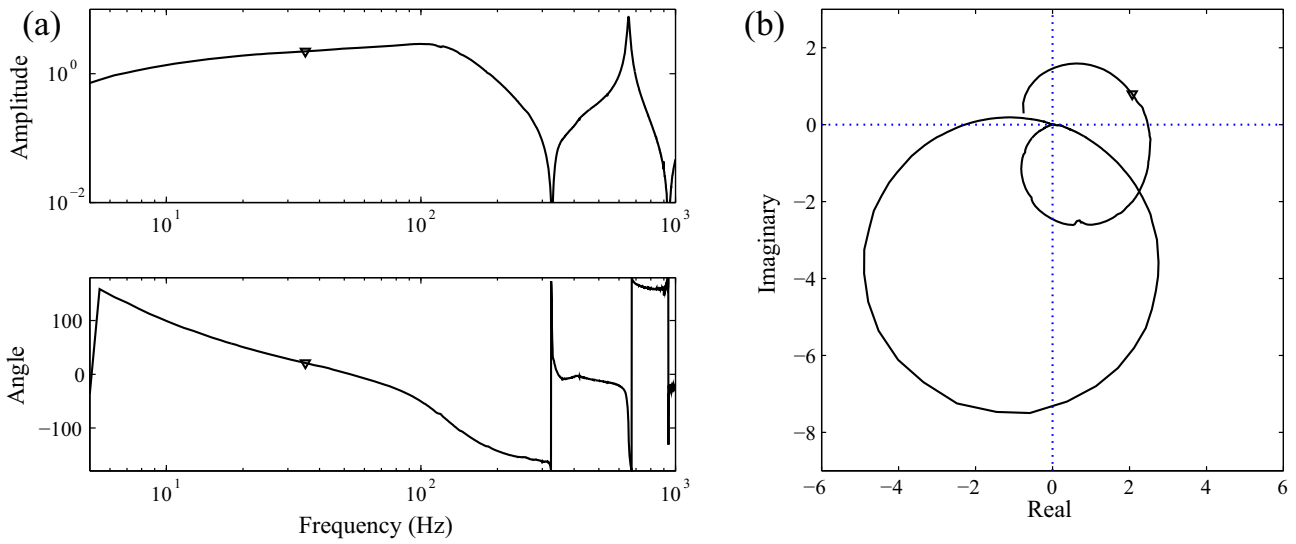

Fig. 3. Plant response in the small cavity: (a) Amplitude (V/V) and phase (degrees) and (b) Nyquist plots.
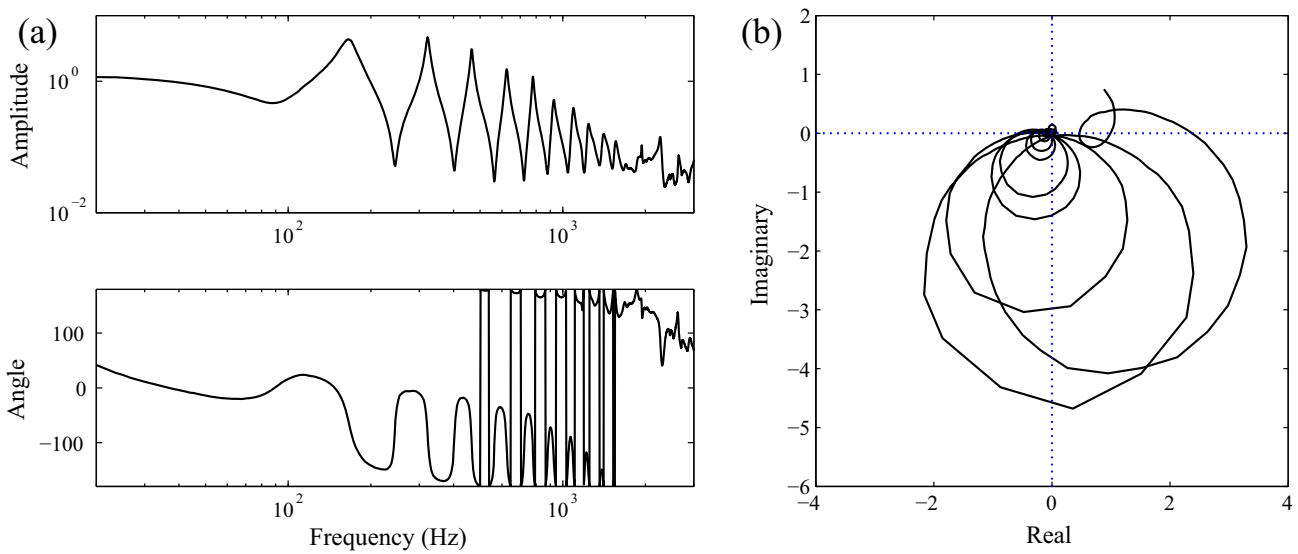

Fig. 4. Plant response in the long duct: (a) Amplitude (V/V) and phase (degrees) and (b) Nyquist plots.

\section{Optimal, robust design framework}

\subsection{Formulation}

Consider the single-input-single-output active noise control system for an acoustic cavity shown in Fig. 5(a). There is a microphone to measure the noise at a target position and is also a loudspeaker to control this noise. The two transducers may or may not be collocated. Primary noise sources are not explicitly shown as they are unimportant in feedback control. The microphone and the loudspeaker are connected via a negative feedback controller $-C(\mathrm{j} \omega)$, where $\mathrm{j}=\sqrt{-1}$ and $\omega$ is the angular frequency. This is disturbance rejection control that can be represented by the general block diagram shown in Fig. 5(b), where $P(\mathrm{j} \omega)$ is the plant and $u(t)$ is the control signal in time $t$. The signals $d(t)$ and $e(t)$ are (a)

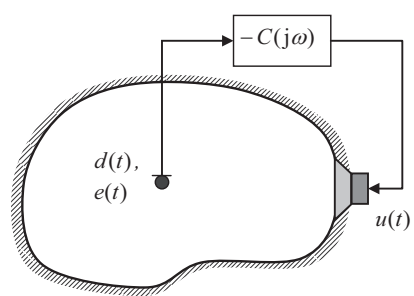

(b)

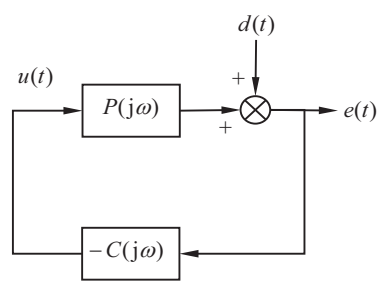

Fig. 5. Disturbance rejection control: (a) schematic drawing of an active noise control system and (b) its block diagram representation. the responses measured by the common error microphone when the control loop is open and closed, respectively. Thus, the two signals cannot be measured simultaneously. Consequently, the block diagram is a representation of a rather mathematical relationship than a real physical circuit. This diagram is used only for mathematical analysis throughout this paper. The performance of the control system can then be specified by the mathematical closed loop FRF or the so-called sensitivity function $S(\mathrm{j} \omega)=e / d$, where $e$ and $d$ are respectively the microphone responses in frequency with and without control. This can be more specifically written as

$S(\mathrm{j} \omega)=[1+L(\mathrm{j} \omega)]^{-1}$,

where $L(\mathrm{j} \omega)=P(\mathrm{j} \omega) C(\mathrm{j} \omega)$ is the open loop FRF. It can also be represented in decibels by $R R(\mathrm{~dB})=20 \log _{10}|S(\mathrm{j} \omega)|$ to practically mean the reduction ratio after control.

With reference to the block diagram in Fig. 5(b), the problem of optimal, robust control can be defined as follows: Given the plant $P(\mathrm{j} \omega)$, find a controller $C(\mathrm{j} \omega)$ that minimizes $|S(\mathrm{j} \omega)|$ in the control bandwidth $\left(\omega \in \Omega_{c}\right)$ under a certain robustness constraint imposed across all frequencies $(\omega \in \Omega)$ such that [16]

$\underset{\omega \in \Omega_{c}}{\operatorname{minimize}}|S(\mathrm{j} \omega)|$,

subject to $|S(\mathrm{j} \omega)| \leqslant 1 / l_{0}, \quad$ (for all frequencies)

where $0<l_{0} \leqslant 1$ and it has been assumed that $P(\mathrm{j} \omega), C(\mathrm{j} \omega)$, and the resulting control system are all stable. Eqs. (2a) and (2b) respectively indicate the performance and the robustness of the control system. In an optimal control state, the inequality constraint in 
Eq. (2b) can be replaced by an equality constraint: $\max |S(\mathrm{j} \omega)|=1 / l_{0}$. This value practically means the maximum allowable control spillover: $R R_{\max }(\mathrm{dB})=-20 \log _{10} l_{0}$, or equivalently the generalized gain margin: $G M(\mathrm{~dB})=-20 \log _{10}\left(1-l_{o}\right)$.

\subsection{Formulation interpretation and the room for uncertainty}

Regarding the robustness of the design framework, it is interesting to know where the value $l_{0}$ in Eq. (2b) comes from. Referring to the Nyquist plot of an open loop FRF illustrated in Fig. 6(a), it is clear that $l_{o}$ indicates the radius of the circle of robustness (dash-dotted line) centered at the instability point $(-1,0)$. From this, it is natural to introduce the generalized gain margin as it has been already defined above. It is further natural to define Nyquist robustness criterion such that a control system is stable and robust with a degree of $l_{o}$ if and only if its open loop FRF locus does not enclose or cross the circle of radius $l_{0}$ centered at the instability point $(-1,0)[16]$. Thus, Eq. (2) is more specifically an optimal, robust design framework based on Nyquist robustness criterion (as well as the sensitivity function $|S(\mathrm{j} \omega)|$ ).

Regarding the performance of the design framework, it is important to understand some general property of the sensitivity function. For most control systems there is a tradeoff called the waterbed effect between the reduction (below unity) and the amplification (above unity) of the sensitivity function $|S(\mathrm{j} \omega)|$, imposed fundamentally by Bode's sensitivity integral [15]. In other words, a reduction in the control bandwidth can in general only be achieved by allowing an increase in some other bandwidth and vice versa. Optimal, robust control is thus a balance between them, achieving large reductions in some important frequency bandwidths while distributing small amplifications in other bandwidths. It is also important to understand the relationship between the open and the closed loop response function given by Eq. (1). It can be seen that for a good performance (i.e., low sensitivity $|S(\mathrm{j} \omega)| \ll 1)$ the open loop response should be large $|L(\mathrm{j} \omega)| \gg 1$ in the control bandwidth, provided that the control system is stable. This condition can be mathematically written as

$\operatorname{Re}[L(\mathrm{j} \omega)] \gg 1 . \quad\left(\omega \in \Omega_{c}\right)$

The case of $\operatorname{Im}[L(\mathrm{j} \omega)] \gg 1$ with $\omega \in \Omega_{c}$ has been excluded since the corresponding locus $L(\mathrm{j} \omega)$ could tend to interfere with the circle of robustness in the range of $\omega \notin \Omega_{c}$. Fig. 6(b) illustrates an open and the corresponding closed loop FRF in decibels. It can be seen that there is a good performance (dashed line) in the control bandwidth at frequencies around $\omega_{c}$ where $|L(\mathrm{j} \omega)|$ (solid line) is large.
There is another issue that is important in practice but has not been incorporated into the mathematical framework given by Eq. (2). This framework is reliable only if the function of $|S(\mathrm{j} \omega)|$ is reliable, which in practice can hardly be met for all frequencies. A reliable strategy to avoid any uncertain attack of instability is thus to make the control effort as small as possible in the unreliable frequency ranges as if there were no feedback action in those ranges. In this regard and generally speaking, the control effort of a practical active control system should be small at very low and very high frequencies. This is because these are often outside the working frequency range, where most acoustic and vibration transducers of piezoelectric type are unreliable and most theoretical plant models can be erroneous. Such small control efforts can then be conveniently assessed by $|L(\mathrm{j} \omega)|$ : a small value of $|L(\mathrm{j} \omega)| \ll 1$ means a weak feedback action. Thus, the plot of $|L(\mathrm{j} \omega)|$ illustrated in Fig. 6(b) is desirable for robustness as the control system works effectively in the control bandwidth while ineffectively at very low and very high frequencies.

From the analyses above, it is clear that the plot of $|S(\mathrm{j} \omega)|$ in Fig. 6(b) can show both the control performance and the control spillover. Whereas, the plot of $|L(\mathrm{j} \omega)|$ can show the robustness to the uncertainty due to immeasurable and unpredictable dynamics. It is thus natural hereby to introduce the room for uncertainty as a robustness measure to this immeasurable uncertainty. As graphically indicated in the plot, this may be defined by the area below the level of $-G M(\mathrm{~dB})$. The room for uncertainty is also a useful measure to judge the relative robustness between controllers, e.g., the electrical damper and the electrical dynamic absorber method compared in [16]. For robustness, not only the circle of robustness but also the room for uncertainty should be taken into consideration. These two are convenient as well as practical concepts as they let a control engineer to conduct control experiments straightforwardly rather than an exhaustive modeling of uncertainty that is partly impossible to model.

\subsection{Comparison}

In the previous two subsections, the optimal, robust design framework based on Nyquist robustness criterion and the room for uncertainty has been constructed by slightly adapting classical $[11]$ and modern $[21,22]$ robust control theory. First, the theories have been tailored to suit disturbance rejection control only, disregarding tracking control. Thus, the sensitivity function has been exclusively used in Eq. (2). Second, the constrained optimization formulation in Eq. (2) is used rather than a standard unconstrained one that involves additional introductions of weighting functions (a)

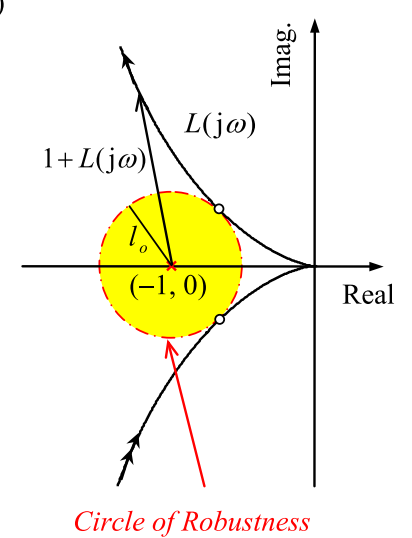

(b)

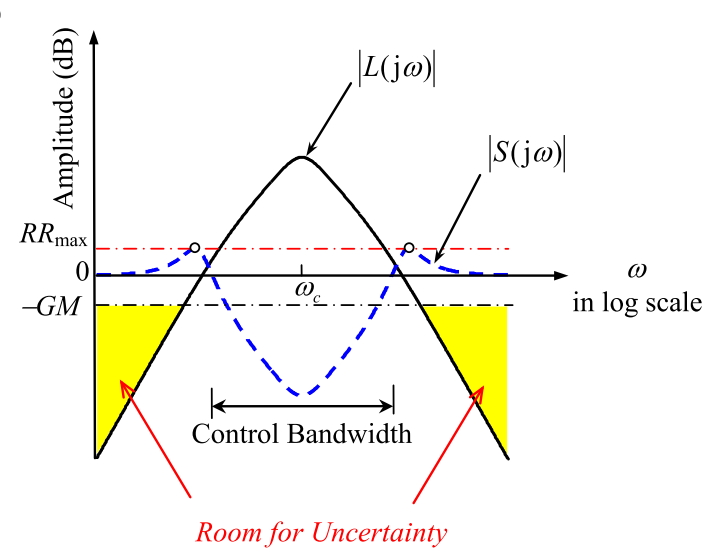

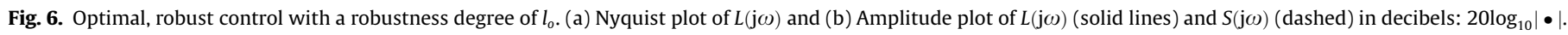
The degree of robustness (dash-dotted) is indicated by a circle of radius $l_{o}$ in (a) and by a level of $R R_{\max }$ in (b). 
accounting for uncertainties. The explicit expression in Eq. (2) is preferred in this paper as the design conducted later in this paper does not rely on any automatic optimization solver but a simple interactive loop shaping technique based on visual inspection. Third, the robustness has been defined by using a single circle centered at the instability point (i.e., the circle of robustness) rather than many different circles along the open loop frequency response points. This has greatly simplified the formulation. Robustness to such frequency dependent uncertainty can instead be separately assessed by visually inspecting $|L(\mathrm{j} \omega)|$ (i.e., the room for uncertainty) in the frequency axis, as explained earlier. Therefore, the design framework in this paper relies on both $S(\mathrm{j} \omega)$ and $L(\mathrm{j} \omega)$ in which the latter is used only for inspecting the room for uncertainty.

As above, the design framework presented here is similar to those in modern robust control theory, but is simpler. It is also similar to the classical design framework based on Nyquist stability criterion combined with the gain and phase margins. Eq. (2) is essentially a compact mathematical description of the classical one, in terms of modern terms (e.g., $l_{0}$ ). Moreover, inspection of the room for uncertainty is analogous to inspection of roll-off characteristics in classical control. Not only the high frequency, but the low frequency roll-off characteristic should be also inspected in general. Thus, it can be stated that the design framework presented in this paper is one that has combined merits of classical and modern robust control theory to suit practical active control of noise and vibration. In this design framework, it should be noted that a proper selection of the fixed-structure controller (i.e., the compensator) is of paramount importance. Some rules and guidelines related are thus discussed in the following section.

\section{Loop shaping technique}

\subsection{Basic rules}

Over decades of endeavors since the introduction of positive position feedback (PPF) in 1990s [12], it has been known that a single second order filter is effective for controlling a single mode in a single narrow frequency bandwidth. It can be also effective for controlling multiple modes in a single broad frequency bandwidth, provided that it is collocated feedback [17]. The former and the latter respectively correspond to the first and the second plant in this paper. Thus, a single second order filter is commonly employed for each of the cases studied. Some basic design rules are addressed in this subsection as to how to determine the type of the filter (e.g., a low, band or high pass filter).

From Eq. (3), a rule of thumb for control performance may be stated as follows:

"Bring the target open loop frequency response point(s) near to the positive real axis of the complex plane"

and make it as large as the robustness in Eq. (2b) allows. Then, the negative feedback architecture will automatically suppress the target response(s) as set by Eq. (1). This rule can be applied to design the controllers for some standard patterns of plant responses often encountered in practical active control of noise and vibration.

As the simplest system, consider the active vibration control of an oscillator consisting of mass $m$, spring $k$ and damper $c$ shown in Fig. 7(a). The position $w$, velocity $\dot{w}$ and acceleration $\ddot{w}$ are individually taken as the output while the force $f$ is the common input. The corresponding plants can be respectively written as

$$
\begin{aligned}
& P_{p}(\mathrm{j} \omega)=(\mathrm{j} \omega)^{-1} \cdot Y, \\
& P_{v}(\mathrm{j} \omega)=Y,
\end{aligned}
$$

$P_{a}(\mathrm{j} \omega)=(\mathrm{j} \omega) \cdot Y$

where $P_{p}(\mathrm{j} \omega)=w / f$ is called receptance, $P_{v}(\mathrm{j} \omega)=\dot{w} / f$ is mobility, $P_{a}(\mathrm{j} \omega)=\ddot{w} / f \quad$ is accelerance, and $Y=c^{-1}\left(\mathrm{j} 2 \zeta_{n} \omega_{n} \omega\right)$ $\left(\omega_{n}^{2}-\omega^{2}+\mathrm{j} 2 \zeta_{n} \omega_{n} \omega\right)^{-1}$ in which $\omega_{n}=\sqrt{k / m}$ is the angular natural frequency and $\zeta_{n}=c /(2 \sqrt{m k})$ is the damping ratio of the oscillator. Note that the three plants are in the forms of a low, band, and high pass filter of second order, respectively. Their responses are shown in Fig. 7(b). It can be seen that the FRF loci reside in the 'lower half, in the 'right half', and in the 'upper half of the complex plane, respectively. Applying the rule of thumb above with $\omega_{n}$ being the target frequency $\omega_{c}$, a possible set of the corresponding control filters of second order can be written as

$$
\begin{aligned}
& C_{p}(\mathrm{j} \omega)=(\mathrm{j} \omega) \cdot Z, \\
& C_{v}(\mathrm{j} \omega)=Z, \\
& C_{a}(\mathrm{j} \omega)=(\mathrm{j} \omega)^{-1} \cdot Z,
\end{aligned}
$$

where $Z=c_{c}\left(\mathrm{j} 2 \zeta_{c} \omega_{c} \omega\right)\left(\omega_{c}^{2}-\omega^{2}+\mathrm{j} 2 \zeta_{c} \omega_{c} \omega\right)^{-1}$ in which $\omega_{c}=\omega_{n}$ is the center frequency and $2 \zeta_{c}$ is the normalized bandwidth. They are now a high, band, and low pass filter of second order, respectively. Their responses are illustrated in Fig. 7(c). These three methods then all yield the same open loop response given by

$L(\mathrm{j} \omega)=P_{i}(\mathrm{j} \omega) \cdot C_{i}(\mathrm{j} \omega)=Y \cdot Z$,

where $i=p, v$ and $a$. This response is shown in Fig. 7(d), where note that the target frequency response (' $\diamond$ ') is in the positive real axis in accord with the rule of thumb. Its magnified view around the circle of robustness has been already shown in Fig. 6(a). Accordingly, those shown in Fig. 6(b) are the corresponding $|L(\mathrm{j} \omega)|$ and $|S(\mathrm{j} \omega)|$.

The examples above have demonstrated that the type of the second order control filter can be determined solely based on the pattern (i.e., receptance, mobility or accelerance) of the plant response in the Nyquist plot. The three equivalent control methods (or basic design rules) above are respectively called positionacceleration feedback (PAF), velocity-velocity feedback (VVF), and acceleration-position feedback (APF) [16]. It is important to note in Fig. 6(b) that they commonly yield the same band pass filter form of $|L(\mathrm{j} \omega)|$, which is desirable for the room for uncertainty. Thus, it can be stated that the three control methods are not only desirable methods for performance but also for robustness. Note here that control filters in desirable forms in the sense of the rule of thumb can be undesirable in the sense of the room for uncertainty, for example, a PPF control filter at low frequencies [12].

Finally, it is interesting to note that many practical plants are, at low frequencies or in a limited band of frequencies, of the three patterns illustrated in Fig. 7(b) [7,8,10,12,14-18,23,24]. For example, the long duct response shown in [7] is similar to that in Fig. 4 revealing a receptance-like response at low frequencies. The headphone response in [10] is similar to that in Fig. 3 revealing a mobility-like response at low frequencies. The ear simulator response in [24] is also in mobility pattern at low frequencies, if inverted. The headset response in [9] is further in mobility pattern at frequencies around $400 \mathrm{~Hz}$, if inverted. The headrest response in [23] is then in accelerance pattern at low frequencies around $230 \mathrm{~Hz}$, if inverted. The vibration plants in [12,14-18] can also be similarly categorized. Thus, the three control methods above can be applied to not only simple oscillators but also to many other practical plants.

\subsection{Guidelines}

Some design guidelines already well known in practical active control are summarized in this subsection. For the active control 
(a)

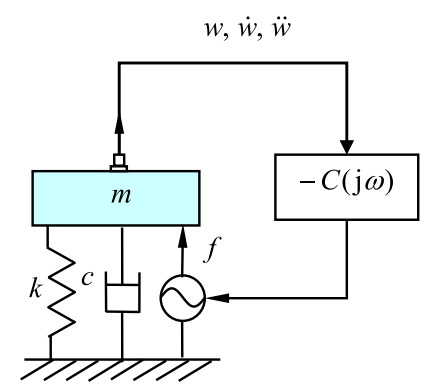

(c)

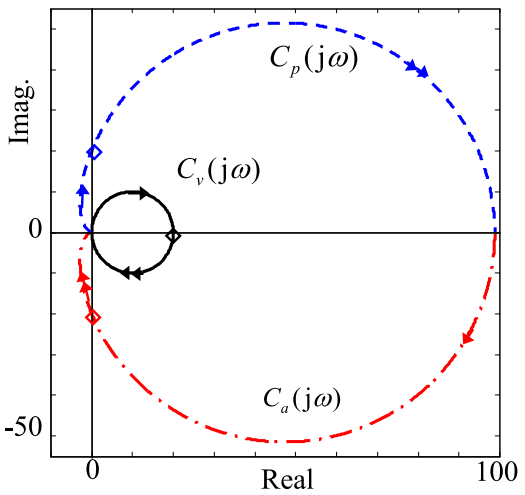

(b)

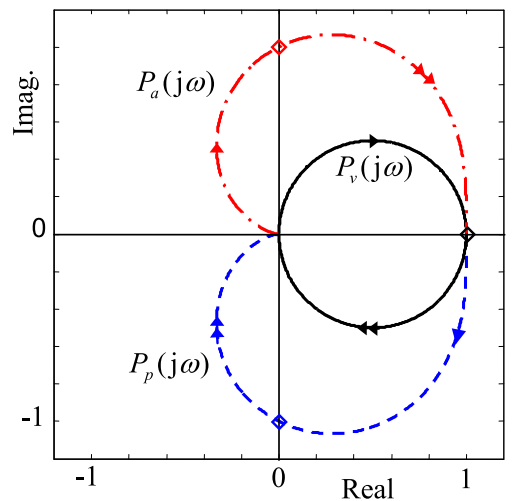

(d)

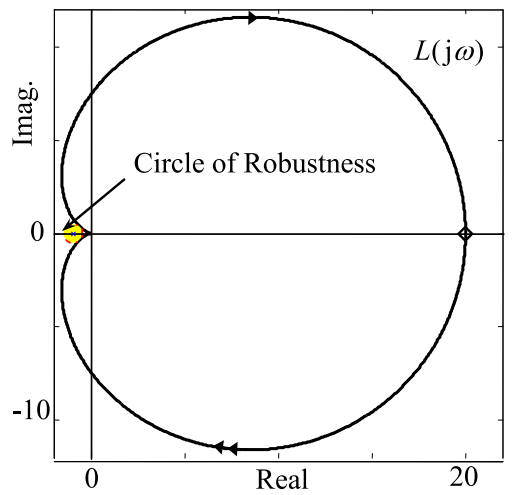

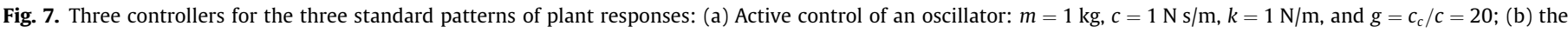

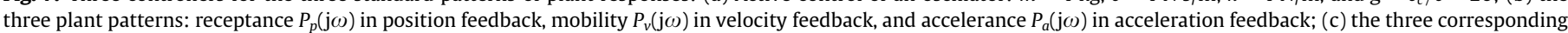

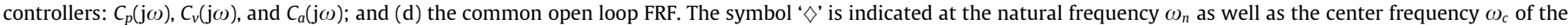
control filter, where $\omega_{n}=\omega_{c}$. The arrows on each locus indicate the direction of increasing frequency.

of cavity noise illustrated in Fig.5(a), the plant response can change its pattern depending on how the loudspeaker behaves in the frequency range of operation, e.g., a spring-, damper- or mass-like behavior [26]. Consequently, a different type of control filter should be employed for each range. If the plant is inverted, the control filter should also be inverted to counteract the effect [18]. Roll-off shaping filters may additionally be introduced to improve the robustness at very low and very high frequencies [17]. The frequency tuning rule $\omega_{c}=\omega_{n}$ already applied earlier is for a single-mode system. It can be slightly altered if the plant is a multi-modal system with considerable mutual interactions between modes. If the concern is now to reduce several modes simultaneously in collocated control, it can be tuned to the center frequency of the bandwidth covering all the target modes. The gain $c_{c}$ and the bandwidth $2 \zeta_{c}$ can then be tuned by loop shaping. In general, the greater the gain $c_{c}$, the greater is the reduction. Further, the greater $c_{c}$ and $2 \zeta_{c}$, the wider is the control bandwidth [16]. If multiple bandwidths are to be controlled, multiple second order filters are generally required each of which is individually designed to control a single individual bandwidth [18]. If there is a considerable time delay in the plant, a phase compensator can be additionally introduced in series with each second order filter [19].

\section{Two designs and experiments}

\subsection{Two designs}

According to the rules in the previous section, the controller for the first plant (the small locus in the 'right half of the complex plane: mobility pattern) shown in Fig. 3(b) should be a band pass filter written as

$C(\mathrm{j} \omega)=c_{c} \frac{\mathrm{j} 2 \zeta_{c} \omega_{c} \omega}{\omega_{c}^{2}-\omega^{2}+\mathrm{j} 2 \zeta_{c} \omega_{c} \omega}$,

where the parameters are later tuned to be $\omega_{c}=2 \pi \times 35 \mathrm{~Hz}$, $c_{c}=62.7$, and $\zeta_{c}=0.005$. Similarly, the controller for the second plant (many loci in the 'lower half' of the complex plane: receptance pattern) shown in Fig. 4(b) should be a high pass filter written as

$C(\mathrm{j} \omega)=\mathrm{j} \omega c_{c} \frac{\mathrm{j} 2 \zeta_{c} \omega_{c} \omega}{\omega_{c}^{2}-\omega^{2}+\mathrm{j} 2 \zeta_{c} \omega_{c} \omega}$,

where the parameters are later tuned to be $\omega_{c}=2 \pi \times 80 \mathrm{~Hz}$, $c_{c}=6.4 e-4$, and $\zeta_{c}=10$. The controller bandwidth of this can be written as $4 \mathrm{~Hz} \leqslant \Omega_{c o n} \leqslant 1604 \mathrm{~Hz}$, where $\left(\omega_{c} / r\right) \leqslant \Omega_{\text {con }} \leqslant\left(\omega_{c} r\right)$ with $r=\left(b+\sqrt{b^{2}+4}\right) / 2$ and $b=2 \zeta_{c}$. The three parameters of each control filter specified above were determined by loop shaping with using the measured plant response. This was performed in a graphical way by repeatedly plotting the open loop FRF locus (as well as $|L(\mathrm{j} \omega)|$ and $|S(\mathrm{j} \omega)|)$ in a computer until a desirable shape was obtained in terms of both performance and robustness. The design procedure for each case can be summarized as follows.

In the first case, the target frequency is $\omega_{t}=2 \pi \times 35 \mathrm{~Hz}$. Step 1: It is desirable to tune the controller center frequency $\omega_{c}$ in Eq. (7) to be $\omega_{t}$ such that

$\omega_{c}=\omega_{t}$

It is then a two-variable tuning problem. Step 2: Either of $\zeta_{c}$ and $c_{c}$ is set to be fixed while the other is changing until a desirable open loop shape is obtained. Step 3: They switch the roles and repeat the process for any improvement. In the second case, the target 
bandwidth is roughly about $165.6 \mathrm{~Hz}$ (1st mode) $\leqslant \Omega_{t} \leqslant 1290 \mathrm{~Hz}$ (8th mode). Step 1: It is desirable to tune the controller bandwidth $\Omega_{\text {con }}$ in Eq. (8) to cover $\Omega_{t}$ such that

$\Omega_{t} \subset \Omega_{\text {con }}$.

Choose a set of $\omega_{c}$ and $\zeta_{c}$ satisfying Eq. (10). It is then a singlevariable tuning problem. Step 2: Increase $c_{c}$ until a desirable open loop shape is obtained. Step 3: Choose many other sets of $\omega_{c}$ and $\zeta_{c}$, and repeat the process for any improvement.

Fig. 8 shows the final desirable open and closed loop FRFs designed for the small cavity under a robustness degree of $l_{o}=1 / 2$. Fig. 9 then shows those for the long duct. The optimal parameters obtained have been already given in Eqs. (7) and (8). It can be seen in each of the Nyquist plots in Figs. 8(a) and 9(a) that the design allows a maximum control spillover of about $6 \mathrm{~dB}$ (the dash-dotted circle). It can be also seen in each amplitude plot that there is a large reduction in the control bandwidth. In the first case shown in Fig. 8(b), there is more than $40 \mathrm{~dB}$ reduction (dashed line) at the target frequency of $35 \mathrm{~Hz}$ with the control bandwidth spanning from $20 \mathrm{~Hz}$ to $75 \mathrm{~Hz}$. In the second case shown in Fig. 9(b), the reductions (dashed line) are respectively about $12 \mathrm{~dB}, 17 \mathrm{~dB}, 16 \mathrm{~dB}$, $13 \mathrm{~dB}, 12 \mathrm{~dB}, 7.3 \mathrm{~dB}, 5.7 \mathrm{~dB}$, and $2.5 \mathrm{~dB}$ for the first eight modes. It is also important to note that each controller designed secures
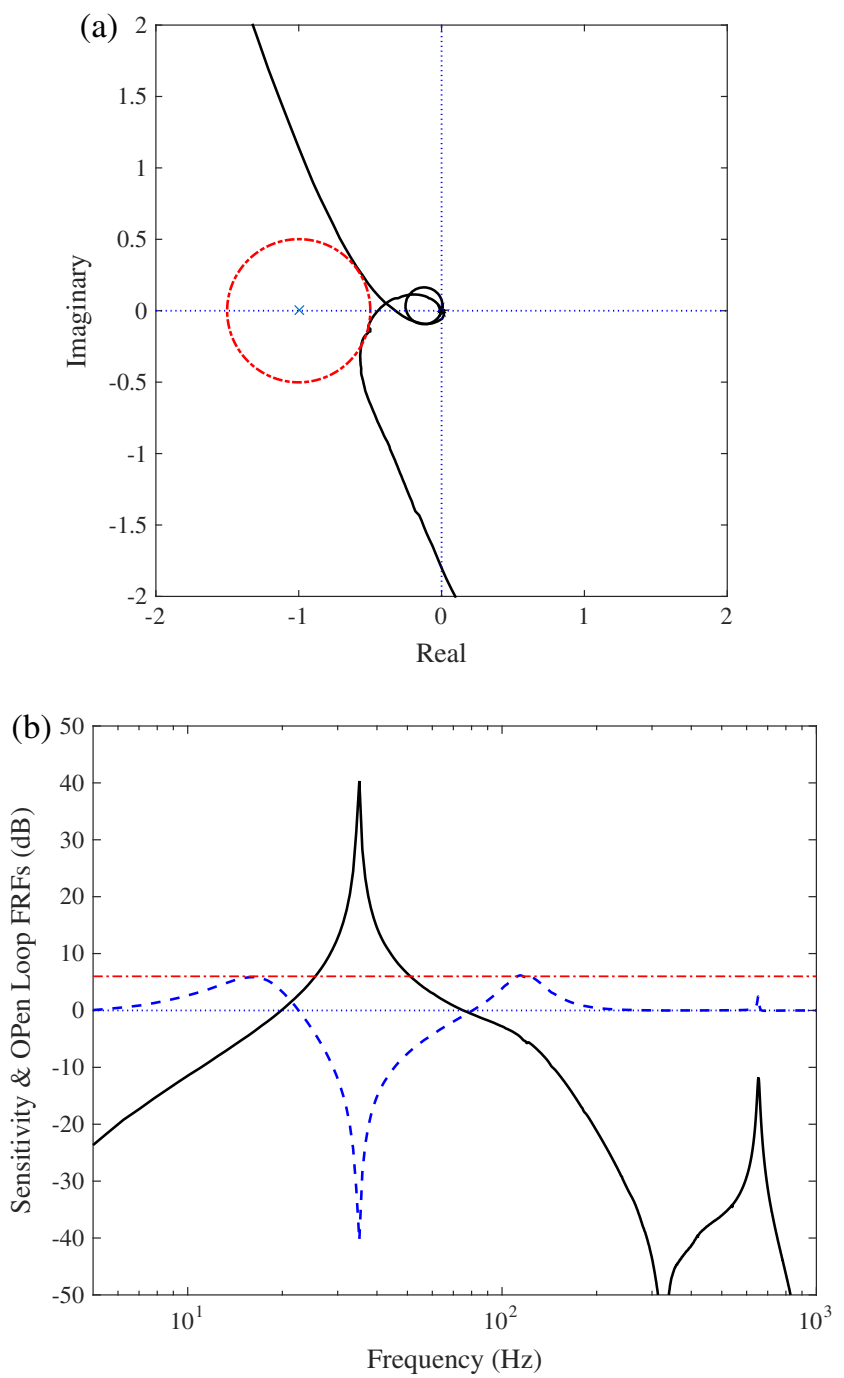

Fig. 8. Controller design for the small cavity with $l_{0}=1 / 2$. Refer to Fig. 6 for detailed captions. large rooms for uncertainty (solid lines) at very low and very high frequencies. It implies that, if implemented in practice, the control systems would be very robust.

\subsection{Experimental results}

The discrete form of each control filter was then implemented within the DSP prototyping machine for real-time control as described in Section 2. The impulse invariant method was used for Eq. (7) [18] while the bilinear transform method ('bilinear' in Matlab $^{\circledR}$ ) was used for Eq. (8) because of the extremely large damping $\zeta_{c}=10$ in this case. A random signal was transmitted to the primary loudspeaker and the microphone signals were measured without and with control (i.e., disconnecting and connecting the control loop, respectively).

Fig. 10 shows the sound pressure responses of the small cavity at the primary and at the control microphone. The performance at the collocated control microphone in (b) is well expected from Fig. 8(b). The large reduction at the remote primary microphone in (a) is also reasonable because the cavity is small and the frequencies are low. Fig. 11 shows those of the long duct. Again, the performance at the collocated control microphone in (b) is well expected from Fig. 9(b). There are also large reductions of the first
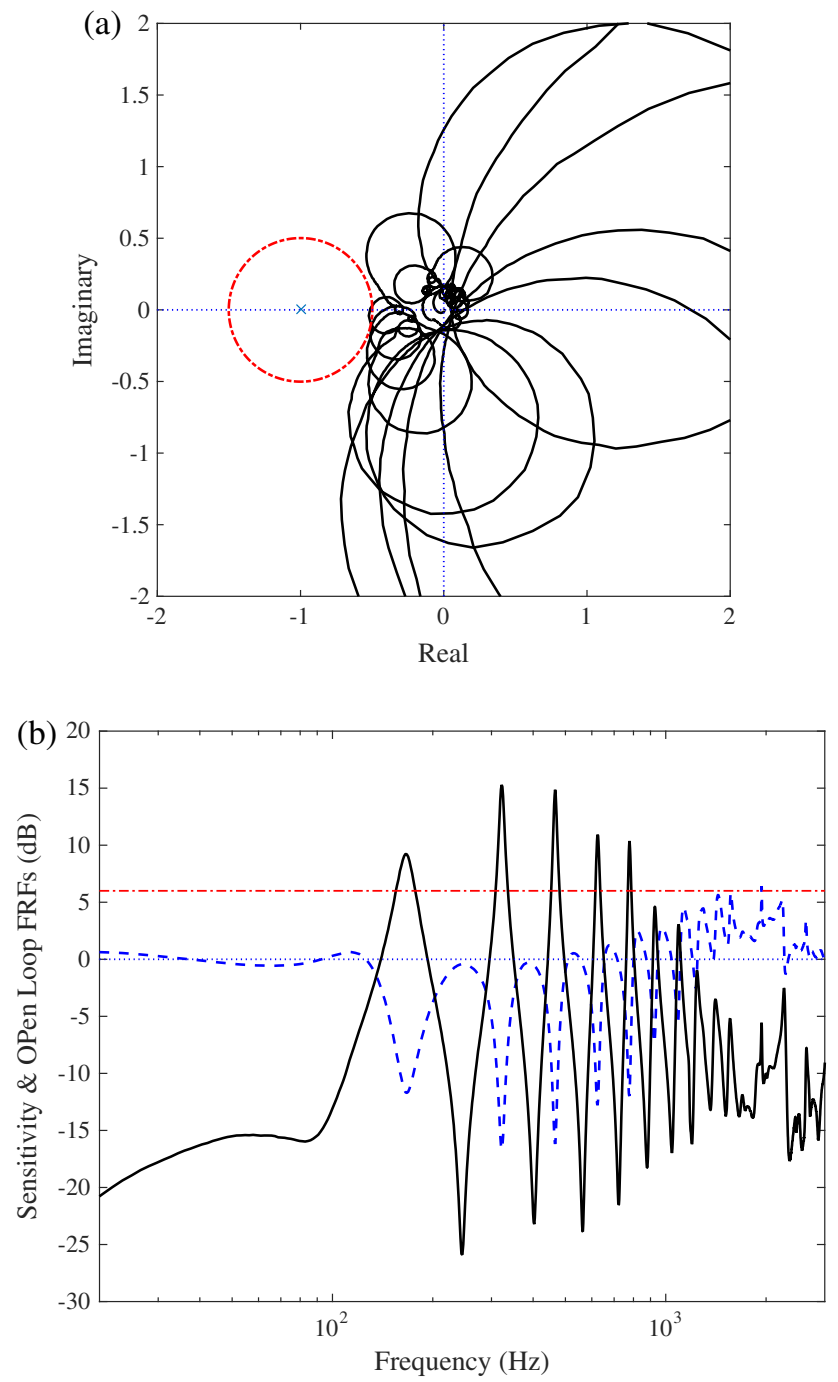

Fig. 9. Controller design for the long duct with $l_{0}=1 / 2$. Refer to Fig. 6 for detailed captions. 

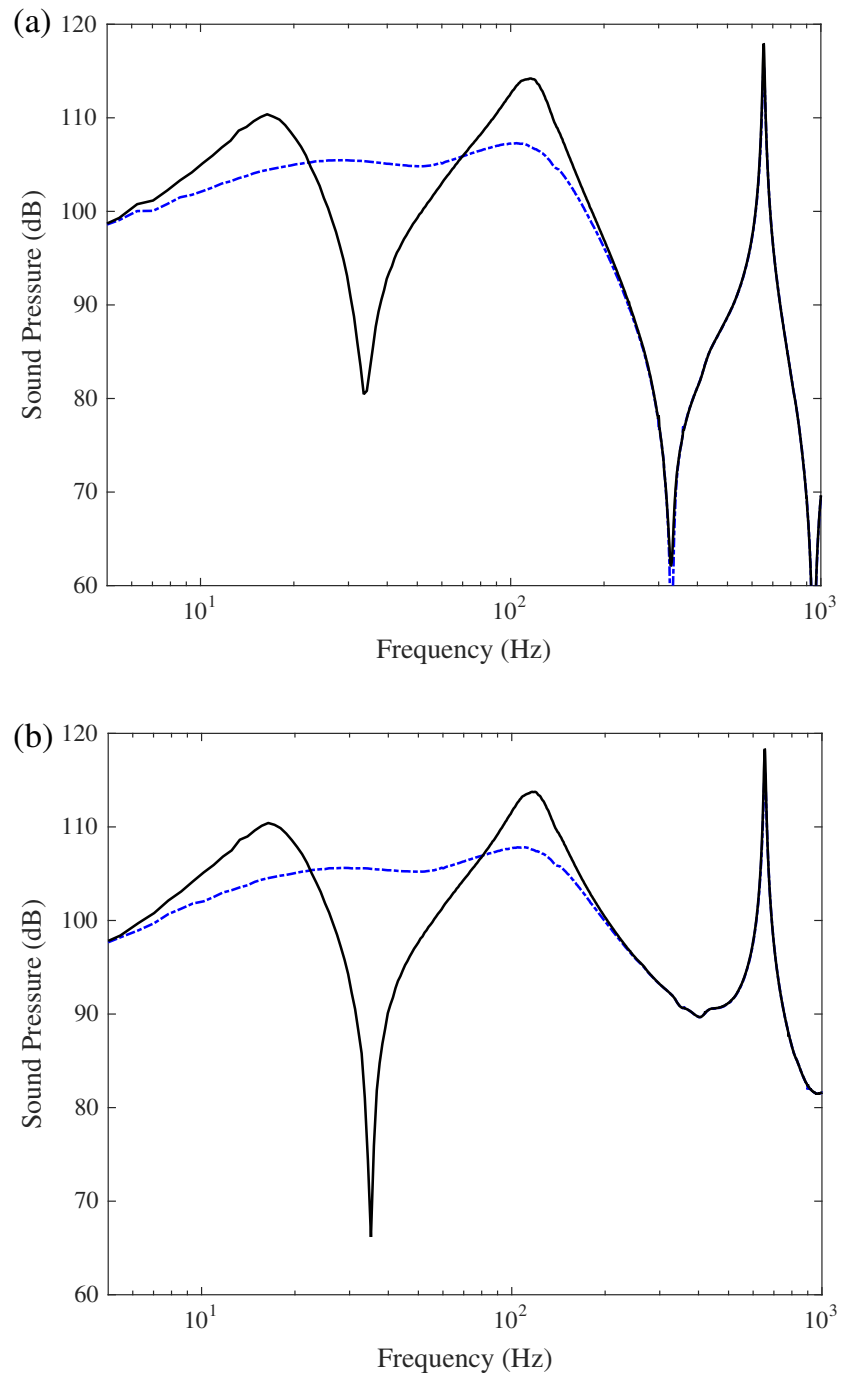

Fig. 10. Sound pressures in the small cavity before (dash-dotted lines) and after (solid) control. (a) Primary microphone $p_{1}$ and (b) control microphone $p_{2}$.

eight modes at the remote primary microphone in (a) and this is also reasonable because it is modal control in such a collocated control configuration [17]. It is noteworthy that there is an enormously large peak at about $2270 \mathrm{~Hz}$ in (a) but not in (b). This is because the primary microphone was positioned at the center but the control microphone was positioned off-center at a nodal point of the first radial mode of the cylindrical duct. This large peak also indicates that the sheet of sound absorbing fabric rolled inside the duct was little helpful in reducing this mode. The results in Figs. 10 and 11 finally suggest that, although it is local feedback using a single loop in each case, it is possible to achieve global control all over the cavity.

\subsection{Discussion}

In the previous subsection, the experimental results of the two cases are displayed. In the first case, a reduction of more than $40 \mathrm{~dB}$ has been achieved in a narrow frequency band around $35 \mathrm{~Hz}$. It is even possible in principle to completely nullify the target tonal noise at the error microphone position [20]. The results here are comparable to those in $[6,10]$. In the second case, the first eight acoustic modes have been simultaneously reduced in a broad frequency band. This is dissimilar to the vibration application in
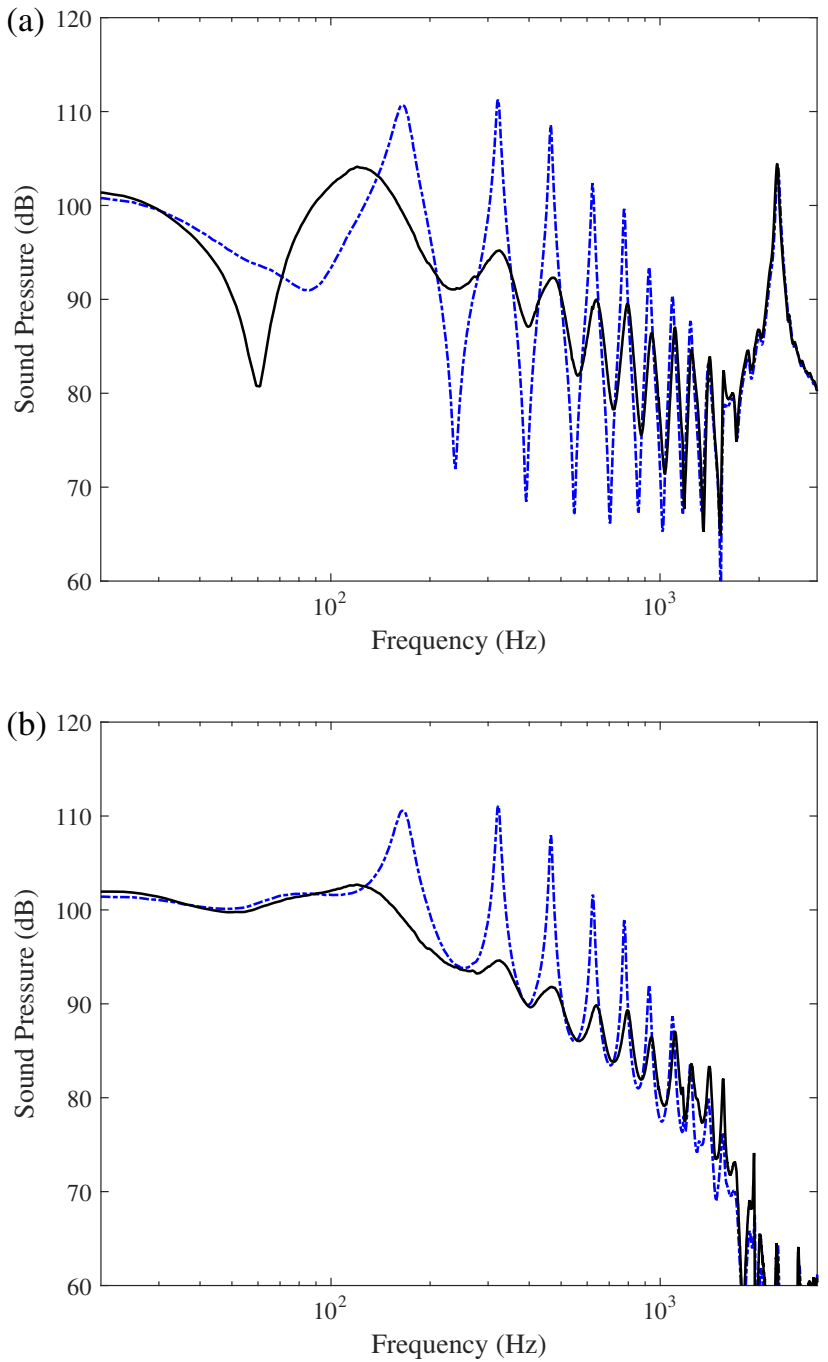

Fig. 11. Sound pressures in the long duct before (dash-dotted lines) and after (solid) control. (a) Primary microphone $p_{1}$ at the center position of the cylinder and (b) control microphone $p_{2}$ at an off-center position.

[17] in that the patterns of the plant responses are different and so are the types of the control filters used. The results here are then comparable to those in [7]. Finally, it should be emphasized that the control performances in this paper have been achieved under a generalized gain margin of $6 \mathrm{~dB}$.

Lastly, it should be emphasized that the two design tasks represented by the plant responses in Figs. 3 and 4 are well-prepared problems after trials and errors, which can thus be easily solved by loop shaping with simple control filters. More specifically, the target frequency of $35 \mathrm{~Hz}$ in Fig. 3(b) resides near the positive real axis in the 'right half of the complex plane, being suitable for applying VVF using a band pass filter of second order. The target frequencies in Fig. 4(b) then reside all near the negative imaginary axis in the 'lower half of the complex plane, being suitable for applying PAF using a high pass filter of second order. It is also important to note that the high pass filter could have been effectively applied since the error microphone was placed at a position where the corresponding plant response gave a good roll-off characteristic at high frequencies (see Fig. 4(a)). Further, the dimensions of the two cavities studied were also carefully chosen. Efforts should thus be devoted to obtain a suitable form of the plant response by any means including relocating transducers, mechanical redesigns of the physical system, applying passive 
damping treatments, etc. As such and also discussed in [27], practical (active noise) control is in general an engineering task involving knowledge and experience of many other disciplines specific to each application (e.g., headphones, compressors, vehicles, etc.) as well as basic control theory. The paper presented here has demonstrated with case studies that the simple data-based loop shaping technique based on visual inspection is a practical tool in this endeavor.

\section{Conclusions}

The two cases studied are narrowband noise control in a small cavity and broadband noise control in a long duct. A simple loop shaping technique has been applied to design the control filter of second order for each case. It is a data-based technique that uses the measured plant response for loop shaping. The parameters of each control filter have been tuned in a graphical way under a well-known design framework based on Nyquist robustness criterion. The room for uncertainty has been additionally inspected to ensure the robustness to immeasurable uncertainty. A discernable aspect of the design process conducted in this paper is that the fixed-structure (i.e., the order and the type) of each control filter has been rather logically determined than a wild guess, solely based on the Nyquist plot of each plant measured. Some rules and guidelines have also been provided to facilitate the said selection as well as the subsequent tuning process of the control filter. Finally, it has been demonstrated with experiments that the technique is practical and a second order filter can be effectively used for active control of cavity noise in a single narrow or broad frequency band.

\section{Acknowledgement}

We thank Mr. C.J. Santana in the Department for Constructing the Experimental Test Rigs.

\section{References}

[1] Guicking D. On the invention of active noise control by Paul Lueg. J Acoust Soc Am 1990;87:2251-4.

[2] Olson HF, May EG. Electronic sound absorber. J Acoust Soc Am 1953;25:1130-6.

[3] Nelson PA, Elliott SJ. Active control of sound. London: Academic Press; 1993.
[4] Kajikawa Y, Gan WS, Kuo SM. Recent advances on active noise control: open issues and innovative applications. APSIPA Trans Signal Inform Process 2012;1:1-21.

[5] Clark RL, Frampton KD, Cole DG. Phase compensation for feedback control of enclosed sound fields. J Sound Vib 1996;195:701-18.

[6] Yu S-H, Hu J-S. Controller design for active noise cancellation headphones using experimental raw data. IEEE/ASME Trans Mechatron 2001;6:483-90.

[7] Bisnette JB, Smith AK, Vipperman JS, Budny DD. Active noise control using phase-compensated, damped resonant filters. J Vib Acoust 2006:128:148-55.

[8] Bao C, Paurobally R, Pan J. Design and test of a feedback controller for attenuating low frequency noise in a room. Acoust Austr 2009;37:61-6.

[9] Zhang L, Wu L, Qiu X. An intuitive approach for feedback active noise controller design. Appl Acoust 2013;74:160-8.

[10] Brandner M, Flock M, Schoerkmaier M. Feedback loop shaping for active noise control with constraints of the worst-case secondary paths. In: 6th congress of alps-adria acoustics association 16-17 October, Graz, Austra.

[11] Ogata K. Modern control engineering. New Jersey: Prentice Hall Inc; 1970.

[12] Fanson JL, Caughey TK. Positive position feedback control for large space structures. AIAA J 1990;28:717-24.

[13] Preumont A. Vibration control of active structures: an introduction. Dordrecht: Kluwer Academic Publishers; 1997.

[14] Pota HR, Moheimani SOR, Smith M. Resonant controllers for smart structures. Smart Mater Struct 2002:11:1-8.

[15] Sethi V, Franchek MA, Song G. Active multimodal vibration suppression of a flexible structure with piezoceramic sensor and actuator by using loop shaping. J Vib Control 2011:17:1994-2006.

[16] Kim SM, Pietrzko S, Brennan MJ. Active vibration isolation using an electrical damper or an electrical dynamic absorber. IEEE Trans Control Syst Technol 2008; $16: 245-54$

[17] Kim SM, Wang S, Brennan MJ. Broadband vibration control of a flexible structure using an electrical dynamic absorber. Smart Mater Struct 2011;20:075002 (9pp).

[18] Kim SM, Oh JE. A modal filter approach to non-collocated vibration control of structures. J Sound Vib 2013;332:2207-21.

[19] Kim SM. Method and apparatus for realizing a generalized electrical dynamic absorber. Korean Patent, 10-1431371; 2014.

[20] Kim SM, Brennan MJ, Abreu GLCM. Narrowband feedback for narrowband control of resonant and non-resonant vibration. Mech Syst Signal Process 2016;76-77:47-57.

[21] Skogestad S, Postlethwaite I. Multivariable feedback control: analysis and design. Chichester: John Wiley \& Sons; 1996.

[22] Gu D-W, Petkov PHr, Konstantinov MM. Robust control design with MATLAB ${ }^{\circledR}$. Leipzig: Springer; 2005.

[23] Rafaely B, Elliott SJ. $\mathrm{H}_{2} / \mathrm{H}_{\infty}$ active control of sound in a headrest: design and implementation. IEEE Trans Control Syst Technol 1999;7:79-84.

[24] Mejia J, Dillon H, Fisher M. Active cancellation of occlusion: an electronic vent for hearing aids and hearing protectors. J Acoust Soc Am 2008;124:235-40.

[25] Kinsler LE, Frey AR, Coppens AB, Danders JV. Fundamentals of acoustics. 4th ed. New York: Wiley; 2000. p. 249-50.

[26] Colloms M, Darlington P. High performance loudspeakers. 6th ed. Chichester: John Wiley \& Sons Ltd; 2005. p. 27-44.

[27] de Oliveira LPR, de Silva MM, Sas P, Van Brussel H, Desmet W. Concurrent mechatronic design approach for active control of cavity noise. J Sound Vib 2008;314:507-25. 